\title{
The significance of ENAH in carcinogenesis and prognosis in gastric cancer
}

\author{
Dan-Dan Wang ${ }^{1, *}$, Qun Jin ${ }^{2, *}$, Lei-Lei Wang ${ }^{3}$, Shu-Fang Han², Yi-Bing Chen ${ }^{4}$, Guo- \\ Dong Sun ${ }^{5}$, Shi-Fei Sun ${ }^{1}$, Shu-Wang Sun ${ }^{1}$, Tao Wang ${ }^{1}$, Fan-Jie Liu ${ }^{1}$, Ping Wang ${ }^{6,7}$ \\ and Bin Shi ${ }^{1}$ \\ ${ }^{1}$ Shandong Medicinal Biotechnology Centre, Key Laboratory for Rare \& Uncommon Diseases of Shandong Province, Back and \\ Neck Pain Hospital of Shandong Academy of Medical Sciences, Jinan 250062, People's Republic of China \\ ${ }^{2}$ The General Hospital of Jinan Military Command, Jinan 250012, People's Republic of China \\ ${ }^{3}$ Key Laboratory for Applied Microbiology of Shandong Province, Ecology Institute of Shandong Academy of Sciences, Jinan \\ 250014, People's Republic of China \\ ${ }^{4}$ Center of Genetic \& Prenatal Diagnosis, First Affiliated Hospital, Zhengzhou University, Zhengzhou 450052, People's \\ Republic of China \\ ${ }^{5}$ Affiliated Hospital of Shandong Academy of Medical Sciences, Shandong Academy of Medical Sciences, Jinan 250031, \\ People's Republic of China \\ ${ }^{6}$ School of Precision Instrument and Opto Electronics Engineering, Tianjin University, Tianjin 300072, People's Republic of \\ China \\ ${ }^{7}$ Shandong Academy of Chinese Medicine, Jinan 250014, People's Republic of China \\ *These authors have contributed equally to this work \\ Correspondence to: Bin Shi, email: sdyky-shibin@163.com \\ Ping Wang, email: wangpingjinan@163.com \\ Keywords: gastric adenocarcinoma, ENAH, expression, prognosis, function \\ Received: February 15, $2017 \quad$ Accepted: July 12, $2017 \quad$ Published: August 02, 2017 \\ Copyright: Wang et al. This is an open-access article distributed under the terms of the Creative Commons Attribution License 3.0 \\ (CC BY 3.0), which permits unrestricted use, distribution, and reproduction in any medium, provided the original author and source \\ are credited.
}

\section{ABSTRACT}

The ENAH gene, which encodes a member of the enabled/vasodilator-stimulated phosphoprotein (Ena/VASP) family of proteins, is involved in the assembly of actin filaments required for cell adhesion and motility. Recent studies show overexpressed ENAH in several cancer types, and ENAH correlates with tumor invasiveness. This study aimed to investigate the expression and function of ENAH in primary gastric adenocarcinoma, and its prognostic significance. We found significantly increased mRNA $(P=0.0283)$ and protein $(P=0.0301)$ expression of ENAH in gastric cancer tissues. ENAH expression markedly associated with tumor size $(P<0.001)$, T stage $(P$ $<0.001)$, N stage $(P=0.001)$, TNM stage $(P<0.001)$ and prognosis $(P<0.001)$. Cox regression analyses revealed ENAH expression as an independent predictor of overall survival $(P=0.019)$. We also analyzed data of 155 gastric cancer cases from The Cancer Genome Atlas (TCGA) and found that ENAH expression significantly correlated with age $(P=0.003)$, T stage $(P=0.023)$ and prognosis $(P=0.05)$. Furthermore, the function of ENAH in cell proliferation, colony formation, cell migration and invasion of gastric cancer cells was analyzed in vitro. Knockdown of ENAH expression suppressed cell proliferation, colony formation, cell migration and invasion in MKN45 cells. Conversely, overexpression of ENAH promoted cell proliferation, cell migration and invasion in MGC803 cells. Our research suggests that ENAH might play promoting functions in carcinogenesis and progression of gastric cancer, and may serve as a valuable prognostic marker for primary gastric adenocarcinoma patients. 


\section{INTRODUCTION}

Gastric cancer (GC) is one of the most common malignances and the leading cause of cancer-related death worldwide [1], with almost 1 million new cases and over 720,000 deaths reported in 2012 [2]. Statistics show that the overall clinical outcome for patients with advanced GC is poor, with 5-year survival rates of only about $5-20 \%$ and a median overall survival of 10 months [2,3]. Despite developments in early detection and treatment (including radical surgery, chemotherapy, and radiotherapy), distant metastasis and local recurrence still occurs in most GC cases, and the clinical outcomes remains far from satisfactory [4-6].

$\mathrm{GC}$ results from a combination of environmental factors and the accumulation of generalized and specific genetic alterations, involving the activation of oncogenes and the inhibition of tumor suppressor genes [7]. Therefore, investigating the genetic alterations and molecular mechanisms involved in gastric carcinogenesis and progression will be critical for improving the diagnosis and treatment of GC [8].

ENAH, a member of the enabled (Ena)/vasodilatorstimulated phosphoprotein (VASP) family, is an actin regulatory protein involved in cell motility and adhesion $[9,10]$. ENAH was recently reported to be up-regulated in many human cancers, including breast cancer and melanoma [11-13]. ENAH expression correlates with tumor grade and vascular invasion in salivary tumor [10]. In addition, ENAH expression is increased in hepatocellular carcinoma and is associated with tumor differentiation and clinical stage [14]. But thus far, the expression, clinical significance, and biological functions of ENAH in GC have not been explored.

In the present study, we analyzed ENAH expression levels in GC samples, and evaluated the functional role of ENAH in the tumorigenesis and progression of primary GC. We further identified the relationship between ENAH expression and clinicopathological features using a large GC population and evaluated its prognostic value in GC.

\section{RESULTS}

\section{ENAH is overexpressed in human GC}

To evaluate the expression pattern of ENAH in GC, we examined mRNA levels by real-time quantitative PCR (qRT-PCR) in 36 human primary GC and matched adjacent normal mucosa tissues. The ENAH mRNA expression level was significantly higher in $22(61.1 \%)$ $\mathrm{GC}$ tissues compared with the adjacent non-tumor tissues $(P=0.0283$, Figure 1A).
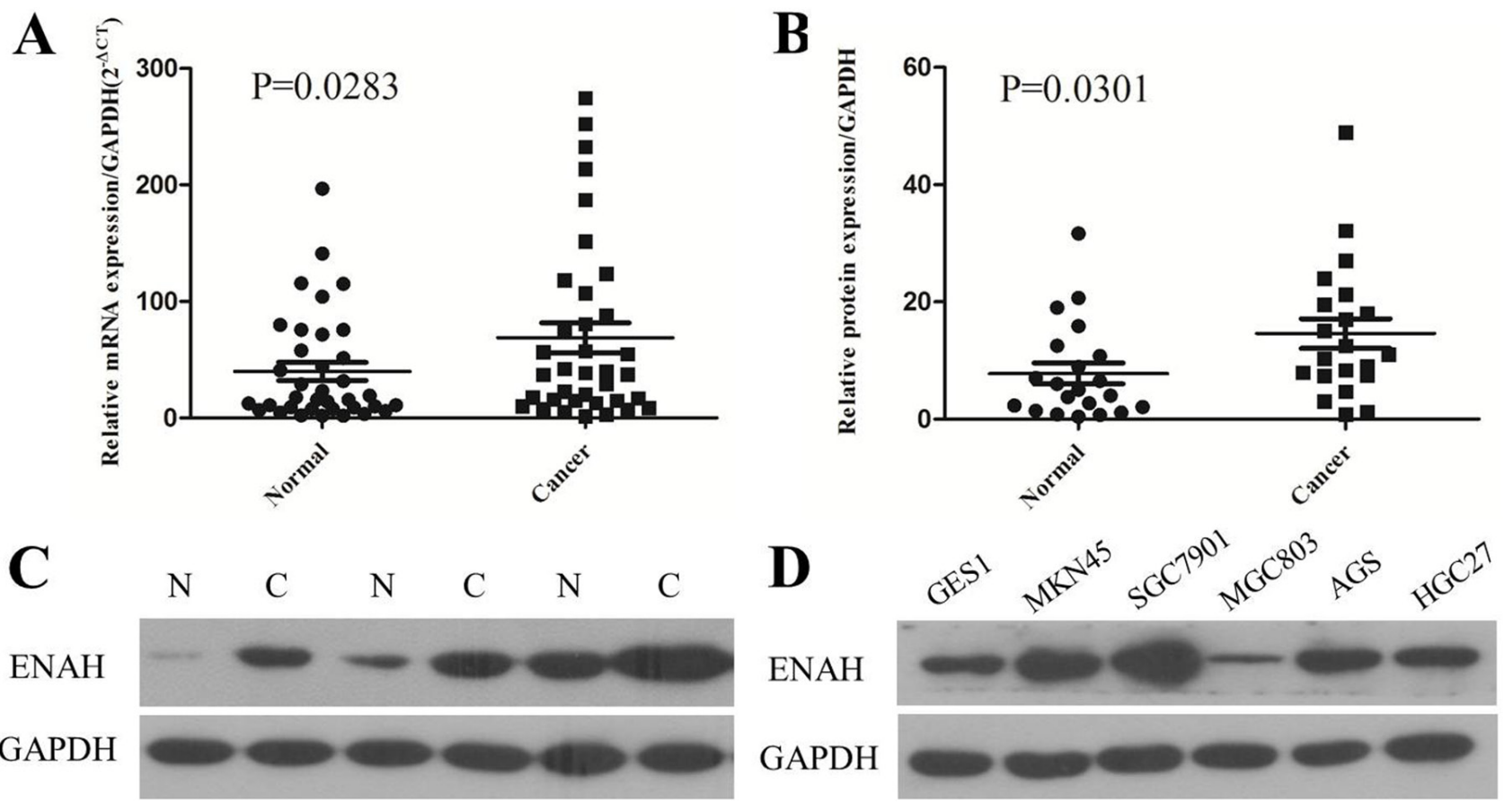

Figure 1: The mRNA and protein expression of ENAH is higher in human primary gastric adenocarcinoma surgical specimens and GC cell lines compared to controls. (A) The relative mRNA expression of ENAH was significantly higher in GC tissues compared with the matched adjacent noncancerous tissues $(\mathrm{n}=36, P=0.0283)$. Horizontal lines represent the mean. (B) Relative ENAH protein expression was significantly higher in GC tissues compared with the matched adjacent noncancerous tissues (ENAH/ GAPDH, $\mathrm{n}=21, P=0.0301$ ). Horizontal lines represent the mean. (C) Representative results of ENAH protein expression in three paired GC tissues and matched adjacent noncancerous tissues ( $\mathrm{N}$, matched noncancerous gastric mucosa; $\mathrm{C}$, GC tissues). (D) ENAH protein expression in the normal gastric cell line GES1 and in GC cell lines MKN45, SGC7901, MGC803, AGS, and HGC27. 
Consistent with the results of qRT-PCR, our western blot analyses revealed higher protein expression of ENAH in $76.2 \%(16 / 21)$ of GC tissues than their matched non-cancerous tissues $(P=0.0301$, Figure $1 \mathrm{~B}$ and $1 \mathrm{C})$. Likewise, the ENAH protein expression was remarkably higher in MKN45, SGC7901, AGS, and HGC27 cell lines compared with the normal gastric cell line GES1 (Figure 1D).

\section{The role of ENAH in proliferation and colony formation in GC cells}

To evaluate the effects of ENAH on cell proliferation, we silenced ENAH expression in the MKN45 cell line with siRNA and then detected the level of ENAH expression in transfected cells by western blotting (Figure 2A). Silencing the expression of ENAH in MKN45 cells significantly inhibited cell proliferation compared with mock siRNA treatment (Figure 2C). Meanwhile, the efficiency of colony formation was significantly $(P=0.0294)$ suppressed in ENAH-specific siRNA transfected GC cells compared with mock siRNA transfected GC cells (Figure 2E and 2F).
ENAH protein expression was lower in MGC803 cells compared with GES1 cells (Figure 1D). Therefore, to further confirm the function of ENAH, we constructed an ENAH expression vector (pDC316-mCMV-ENAH) and transfected MGC803 cells (Figure 2B). The cell growth rate was significantly enhanced in MGC803 cells overexpressing ENAH (Figure 2D).

\section{ENAH promotes cell migration and invasion in GC cells}

We conducted transwell assay to evaluate the effects of ENAH expression on GC cell migration and invasion. Silencing ENAH expression in MKN45 cells resulted in a significant decrease in the number of cells that migrated or invaded through the membrane in the transwell chamber (Figure 3A and 3B). Conversely, overexpression of ENAH significantly enhanced the migration and invasion of MGC803 cells compared with MGC803 cells transfected with a control vector (Figure 3C and 3D). These results suggest that increased ENAH expression may further promote GC progression.

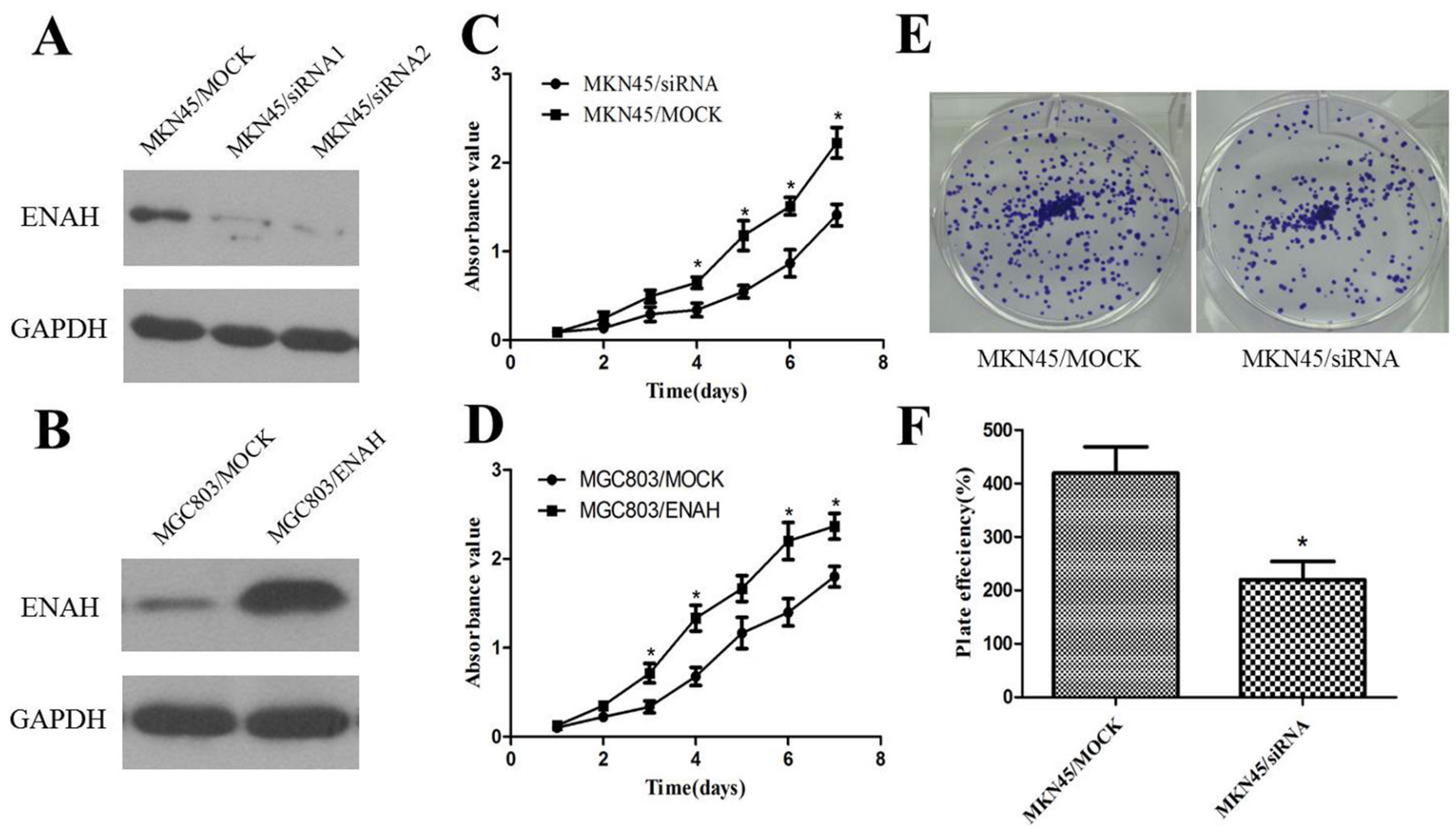

Figure 2: ENAH promotes proliferation and colony formation in MKN45 and MGC803 cell lines. (A) Western blot analysis of silenced ENAH expression in MKN45 cells. (B) Western blot analysis of ENAH overexpression in MGC803 cells. (C) Cell-proliferation assay showing that silencing ENAH expression inhibited proliferation of MKN45 cells. (D) Cell-proliferation assay showing that ENAH overexpression promoted proliferation of MGC803 cells. (E) Representative results showing silencing ENAH expression inhibited colony formation of MKN45 cells. (F) Quantitative analyses of foci numbers in Figure 2E shown as the mean $\pm \mathrm{SD}$. $* P<0.05$ versus control. 
A

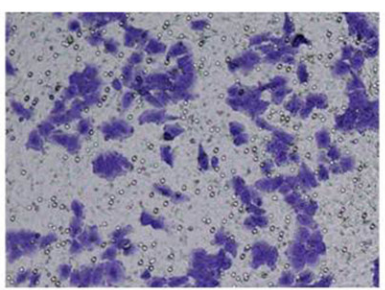

MKN45/MOCK
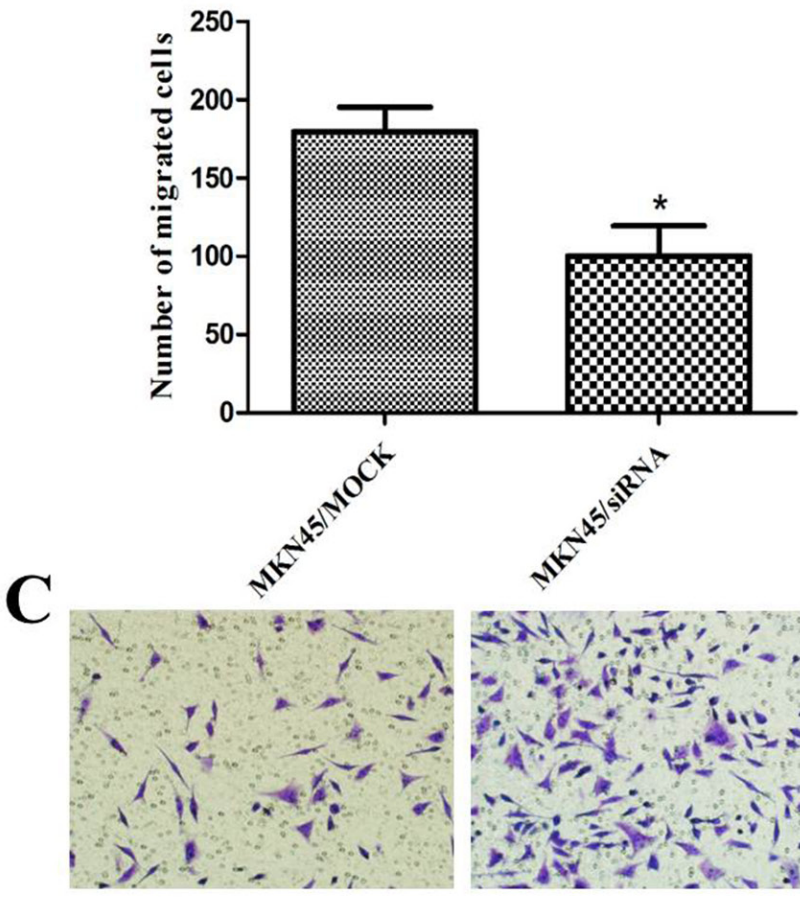

MGC803/MOCK
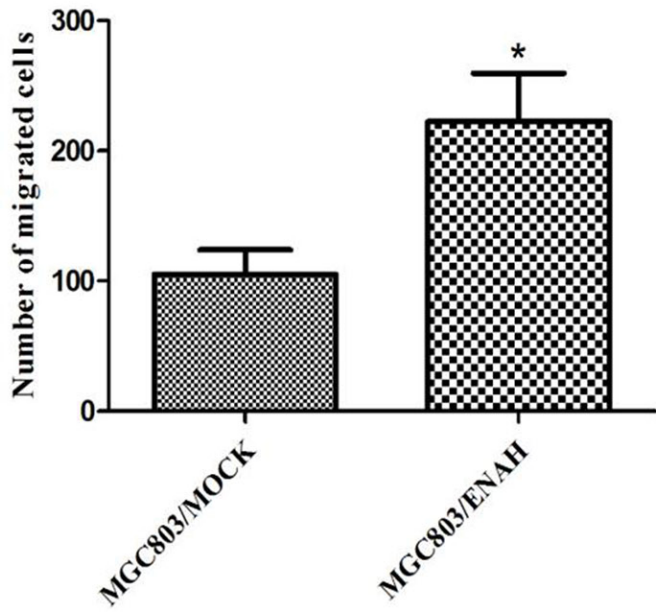

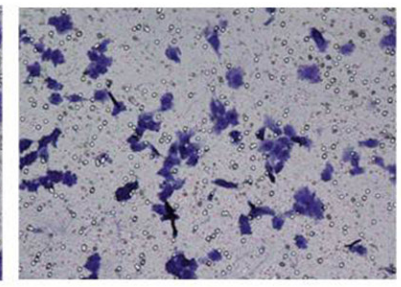

MKN45/siRNA
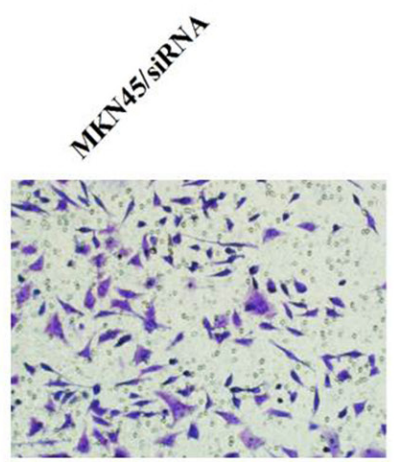

MGC803/ENAH

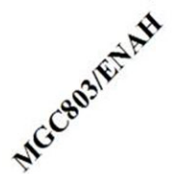

B

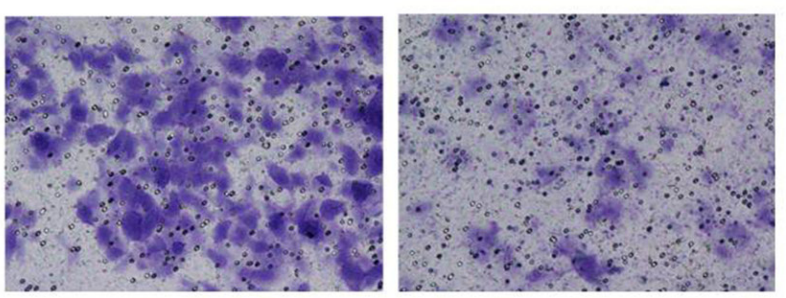

MKN45/MOCK

MKN45/siRNA
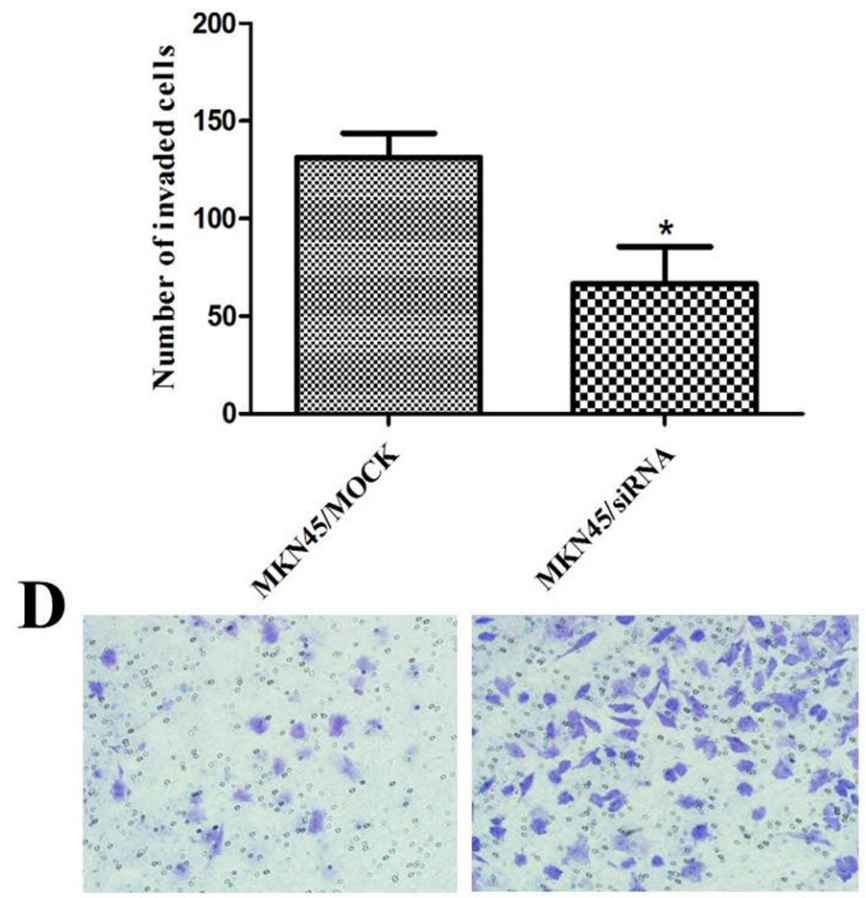

MGC803/MOCK MGC803/ENAH

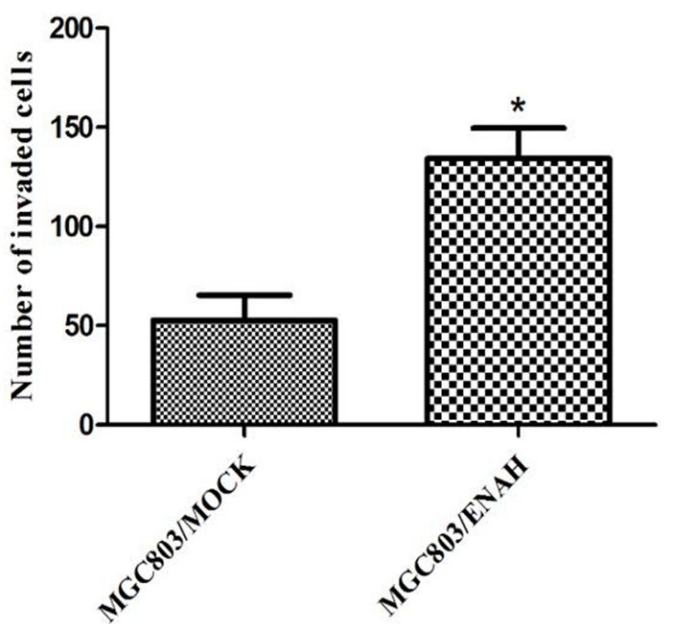

Figure 3: ENAH promotes cell migration and invasion in MKN45 and MGC803 cells. Images (upper panel) of the transwell migration and matrigel invasion assays are shown using $\times 100$ magnification; ten randomly selected fields were used for quantification (lower panel, bar graphs). Values are expressed as the mean $\pm \mathrm{SD}$ of three independent experiments. (A, B) ENAH knockdown inhibited migration (A) and invasion (B) of MKN45 cells. (C, D) ENAH overexpression promoted migration (C) and invasion (D) of MGC803 cells. $* P<0.05$ versus control. 


\section{Immunohistochemical analysis of ENAH} expression in GC tissue samples and its relationship with clinicopathological parameters

To further elucidate the clinicopathological and prognostic roles of ENAH expression, we carried out immunohistochemical analyses of the 238 paraffinembedded GC tissues. Among the $238 \mathrm{GC}$ tissue samples, we found $102(42.9 \%)$ cases with low ENAH expression and $136(57.1 \%)$ cases with high ENAH expression. Noncancerous gastric mucosa and well-differentiated gastric adenocarcinoma showed low ENAH expression (Figure 4A and 4B). Moderately and poorly differentiated gastric adenocarcinoma showed high ENAH expression (Figure 4C and 4D).

Chi-square analyses revealed that ENAH expression was significantly correlated with tumor size $(P<0.001)$, depth of tumor infiltration (T stage, $P<0.001$ ), local lymph node metastasis (N stage, $P=0.001$ ), and TNM stage $(P<0.001)$, but not with age, gender, and distant metastasis (M stage). The correlations between ENAH expression and clinicopathological parameters are listed in Table 1.

\section{ENAH expression and patient survival}

The 5-year overall survival rates in patients with low and high ENAH expression were $71.5 \%$ and $47.6 \%$, respectively. Kaplan-Meier analyses revealed that the overall survival of GC patients with high ENAH expression was significantly worse than that of patients with low ENAH expression $(P<0.001$, log-rank test, Figure 5A).

\section{Univariate and multivariate analyses of prognostic variables in GC patients}

Further univariate and multivariate analyses were performed using a Cox proportional-hazards model to investigate the effect of ENAH expression and
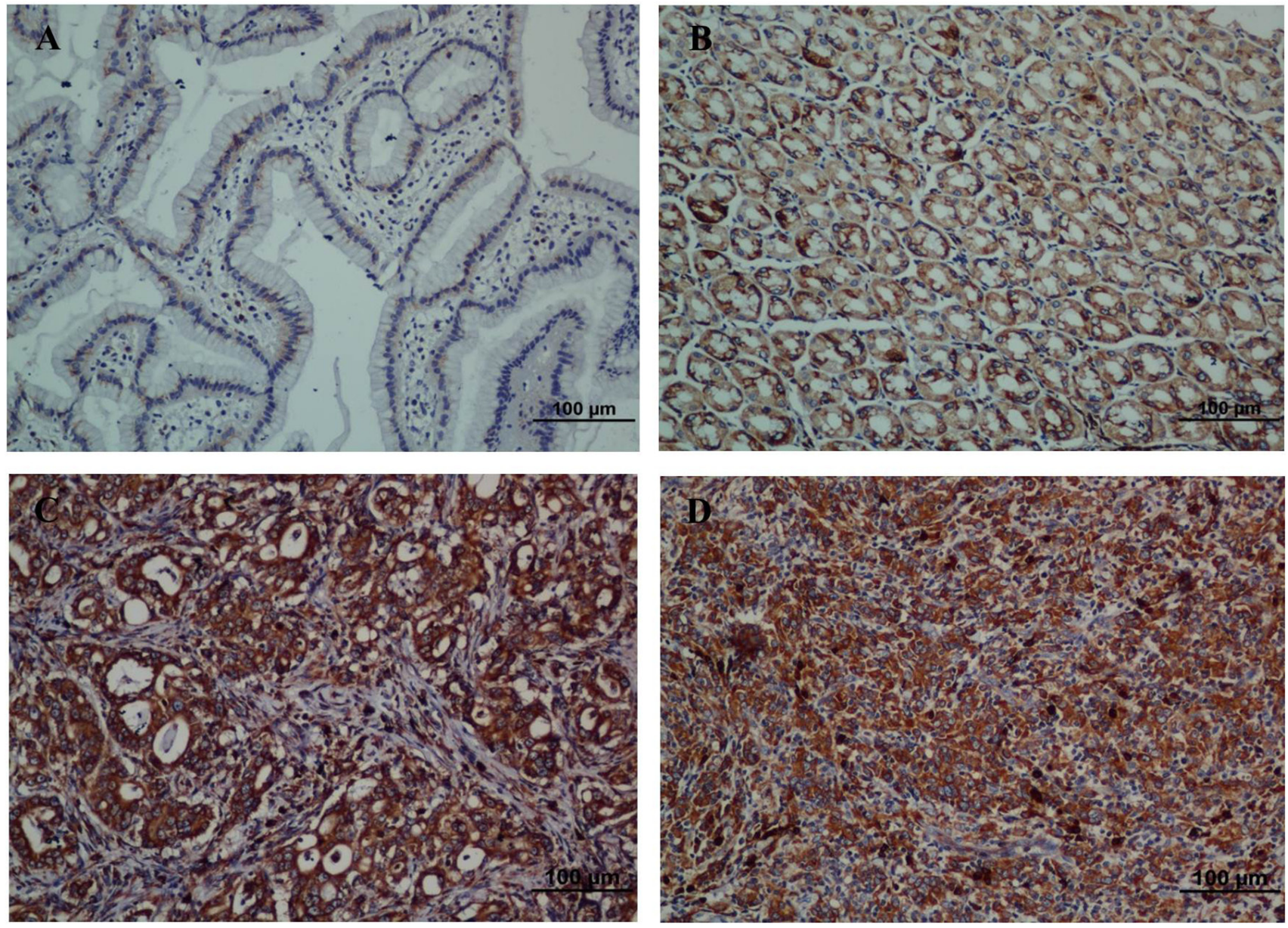

Figure 4: Strong expression of ENAH protein is observed in GC surgical specimens by immunohistochemistry. (A) Weak ENAH staining was observed in noncancerous gastric mucosa. (B) Weak ENAH staining was observed in well-differentiated GC. (C) Strong ENAH staining was observed in moderately differentiated GC. (D) Strong ENAH staining was observed in poorly differentiated GC. 
Table 1: Correlation between ENAH expression and clinicopathological variables of 238 gastric cancer cases

\begin{tabular}{|c|c|c|c|c|c|}
\hline \multirow{2}{*}{ Clinicopathological parameters } & \multirow{2}{*}{$n^{\mathrm{a}}$} & \multicolumn{2}{|c|}{ ENAH expression } & \multirow{2}{*}{$\chi^{2}$} & \multirow{2}{*}{$P$ value } \\
\hline & & High & Low & & \\
\hline All & 238 & 136 & 102 & & \\
\hline \multicolumn{6}{|l|}{ Age (years) } \\
\hline$<55$ & 116 & 65 & 51 & 0.114 & 0.736 \\
\hline$\geq 55$ & 122 & 71 & 51 & & \\
\hline Gender & & & & 1.021 & 0.312 \\
\hline Male & 87 & 46 & 41 & & \\
\hline Female & 151 & 90 & 61 & & \\
\hline Tumor size & & & & 15.025 & $<0.001^{*}$ \\
\hline$<3 \mathrm{~cm}$ & 51 & 17 & 34 & & \\
\hline$\geq 3 \mathrm{~cm}$ & 187 & 119 & 68 & & \\
\hline Tumor infiltration & & & & 39.028 & $<0.001^{*}$ \\
\hline $\mathrm{T} 1$ & 41 & 10 & 31 & & \\
\hline $\mathrm{T} 2$ & 37 & 13 & 24 & & \\
\hline $\mathrm{T} 3$ & 4 & 2 & 2 & & \\
\hline T4a & 111 & 82 & 29 & & \\
\hline $\mathrm{T} 4 \mathrm{~b}$ & 45 & 29 & 16 & & \\
\hline Local lymph node metastasis & & & & 16.250 & $0.001 *$ \\
\hline No & 84 & 36 & 48 & & \\
\hline N1 & 44 & 23 & 21 & & \\
\hline N2 & 37 & 23 & 14 & & \\
\hline N3 & 73 & 54 & 19 & & \\
\hline Distant metastasis & & & & 0.015 & 0.903 \\
\hline M0 & 213 & 122 & 91 & & \\
\hline M1 & 25 & 14 & 11 & & \\
\hline TNM staging & & & & 40.926 & $<0.001 *$ \\
\hline 1 & 48 & 9 & 39 & & \\
\hline 2 & 96 & 61 & 35 & & \\
\hline 3 & 65 & 50 & 15 & & \\
\hline 4 & 29 & 16 & 13 & & \\
\hline
\end{tabular}

${ }^{\text {a }}$ Numbers of cases in each group. $*$ Statistically significant $(P<0.05)$.

other clinicopathological parameters in GC patients. Univariate Cox regression analyses showed that tumor size $(P<0.001)$, T stage $(P<0.001), \mathrm{N}$ stage $(P<$ $0.001)$, M stage $(P<0.001)$, TNM stage $(P<0.001)$ and ENAH expression $(P<0.001)$ were significant prognostic factors (Table 2). A multivariate Cox regression analysis confirmed tumor size $(P=0.036)$, TNM stage $(P<0.001)$, and ENAH expression $(P=$
0.019 ) as independent prognostic predictors for GC patients (Table 2).

\section{Clinicopathological and prognostic analyses with} TCGA clinical GC data

We analyzed data of 155 TCGA GC cases downloaded from the UCSC Cancer Genomics Browser 
database. Among the 155 TCGA GC cases, we found $67(43.2 \%)$ cases with low ENAH expression and 88 (56.8\%) cases with high ENAH expression (Table 3). ENAH expression was significantly correlated with depth of tumor infiltration (T stage, $P=0.023$, Table 3 ) and age $(P=0.003$, Table 3$)$.

Kaplan-Meier analyses revealed that the overall survival of GC patients with high ENAH expression was significantly worse than that of patients with low ENAH expression $(P=0.05$, log-rank test, Figure $5 \mathrm{~B})$. Univariate Cox regression analyses showed that $\mathrm{N}$ stage $(P=0.049)$, M stage $(P=0.002)$, and TNM stage $(P=0.042)$ were significant prognostic factors, and the $P$ value for ENAH expression was 0.056 (Table 4$)$. TNM stage $(P=0.037)$ was confirmed as a significant independent predictor by multivariate Cox regression analysis (Table 4).

\section{DISCUSSION}

The Ena/VASP family comprises three members: Ena-VASP-like (Evl), Mena (ENAH), and VASP. These proteins have similar tripartite domain organization consisting of an N-terminal Ena/VASP homology 1 (EVH1) domain and a variable central proline-rich region, followed by a C-terminal EVH2 domain [15, 16]. The EVH1 domain is responsible for targeting Ena/ VASP proteins to the leading edge of cells to control cell migration and motility, and the $\mathrm{EVH} 2$ domain regulates actin polymerization [17].

A

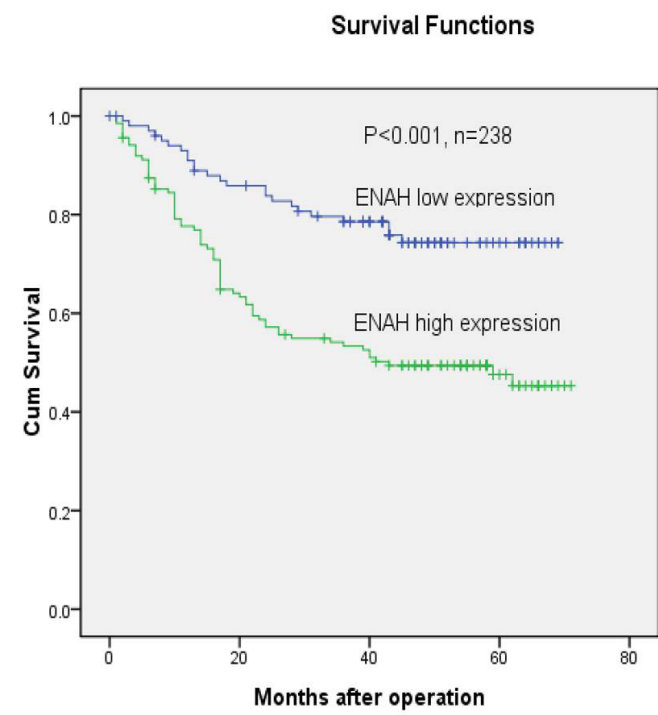

While ENAH expression was recently reported to be increased in breast cancer, hepatocellular carcinoma, and melanoma [10-13], its role in GC remained undefined. In the current study, we found mRNA and protein expression of ENAH were significantly higher in primary GC tissues compared with adjacent non-tumor tissues. Likewise, the ENAH protein expression was remarkably higher in MKN45, SGC7901, AGS and HGC27 cell lines, compared with normal gastric cell line GES1. ENAH was also found to be highly expressed in 136 out of 238 (57.1\%) GC samples, with lower expression in another 102 (42.9\%) cases. Finally, analysis of 155 samples from the TCGA databases revealed 67 of $155(43.2 \%)$ GC samples with low ENAH expression and 88 (56.8\%) cases with high ENAH expression.

We then explored the role of ENAH in the proliferation and colony formation of GC in vitro. We found that silencing ENAH expression in MKN45 cells significantly inhibited cell proliferation and colony formation, whereas ENAH overexpression in MGC803 cells significantly enhanced cell growth rate and colony formation. These results indicated that ENAH may promoting tumor cell growth.

ENAH protein has been shown to have several splice variants, including the $\mathrm{hMena}^{\mathrm{INV}}$ and hMena ${ }^{11 \mathrm{a}}$ isoforms [18-20]. The $\mathrm{hMena}^{\mathrm{INV}}$ isoform is reportedly expressed exclusively in invasive cancer cells [19, 20], while the $\mathrm{hMena}^{1 \mathrm{la}}$ isoform is expressed specifically in epithelia in primary breast carcinomas and is downregulated in invasive tumor cells $[19,20]$. Tanaka et
B

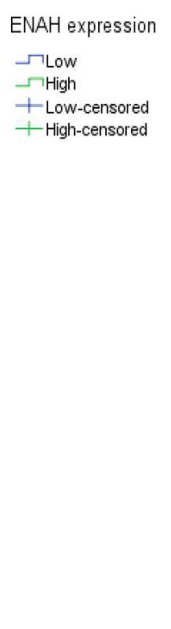

Survival Functions

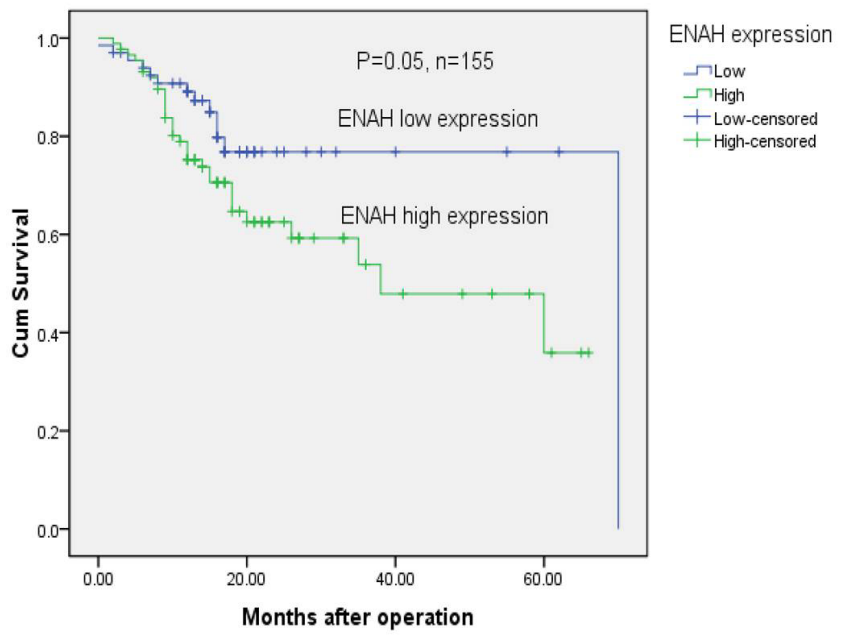

Figure 5: GC patients with high ENAH show lower survival than those with low expression. (A) Kaplan-Meier survival curves of GC patients $(n=238)$ after gastrectomy. The survival rate of patients in high ENAH expression group was significantly lower than that of patients in low ENAH expression group (log-rank test, $P<0.001$ ). (B) Kaplan-Meier survival curves for the TCGA gastric cancer cases $(n=155)$. The survival rate of patients in high ENAH expression group was significantly lower than that of patients in low ENAH expression group (log-rank test, $P=0.05$ ). 
Table 2: Univariate and multivariate analyses of overall survival of gastric cancer patients

\begin{tabular}{|c|c|c|c|c|c|c|c|}
\hline \multirow{2}{*}{ Variables } & \multirow{2}{*}{$n^{\mathrm{a}}$} & \multicolumn{3}{|c|}{ Univariate analyses } & \multicolumn{3}{|c|}{ Multivariate analyses } \\
\hline & & HR & $(95 \% \mathrm{CI})$ & $P$ value & HR & $(95 \% \mathrm{CI})$ & $P$ value \\
\hline Age (years) & & & & 0.217 & & & 0.187 \\
\hline$<55$ & 116 & 1.000 & & & 1.000 & & \\
\hline$\geq 55$ & 122 & 1.295 & $0.859-1.953$ & & 1.330 & $0.870-2.033$ & \\
\hline Gender & & & & 0.468 & & & \\
\hline Male & 87 & 1.000 & & & 1.000 & & 0.923 \\
\hline Female & 151 & 1.170 & $0.765-1.790$ & & 1.022 & $0.660-1.584$ & \\
\hline Tumor size & & & & $<0.001 *$ & & & $0.036^{*}$ \\
\hline$<3 \mathrm{~cm}$ & 51 & 1.000 & & & 1.000 & & \\
\hline$\geq 3 \mathrm{~cm}$ & 187 & 9.835 & $3.112-31.083$ & & 3.633 & $1.088-12.126$ & \\
\hline Tumor infiltration & & & & $<0.001^{*}$ & & & \\
\hline $\mathrm{T} 1-2$ & 78 & 1.000 & & & & & \\
\hline T3-4 & 160 & 19.562 & $6.188-61.837$ & & & & \\
\hline $\begin{array}{l}\text { Local lymph node } \\
\text { metastasis }\end{array}$ & & & & $<0.001 *$ & & & \\
\hline N0 & 84 & 1.000 & & & & & \\
\hline N1 & 44 & 4.452 & $1.815-10.921$ & & & & \\
\hline $\mathrm{N} 2$ & 37 & 6.940 & $2.898-16.619$ & & & & \\
\hline N3 & 73 & 13.052 & $5.923-28.760$ & & & & \\
\hline Distant metastasis & & & & $<0.001 *$ & & & \\
\hline M0 & 213 & 1.000 & & & & & \\
\hline M1 & 25 & 5.397 & $3.301-8.823$ & & & & \\
\hline TNM staging & & & & $<0.001^{*}$ & & & $<0.001^{*}$ \\
\hline $1-2$ & 144 & 1.000 & & & 1.000 & & \\
\hline $3-4$ & 94 & 6.222 & $3.898-9.931$ & & 4.339 & $2.655-7.090$ & \\
\hline ENAH & & & & $<0.001^{*}$ & & & $0.019 *$ \\
\hline Low & 102 & 1.000 & & & 1.000 & & \\
\hline High & 136 & 2.566 & $1.612-4.086$ & & 1.764 & $1.098-2.835$ & \\
\hline
\end{tabular}

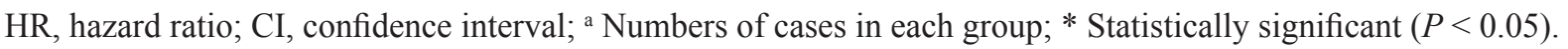

al. found that $\mathrm{hMena} \mathrm{a}^{\mathrm{INV}}$ expression is augmented during tumor progression in breast cancer, and the relative expression of hMena ${ }^{1 \mathrm{la}}$ compared with $\mathrm{hMena}^{\mathrm{INV}}$ is linked to malignant transformation in breast epithelial cells and cancer progression [10]. Therefore, in the present study, we explored the role of ENAH in cell migration and invasion in GC cells. Silencing ENAH expression significantly inhibited the migration and invasion in MKN45 cells, while ENAH overexpression significantly enhanced the migration and invasion in MGC803 cells. Furthermore, immunohistochemical analyses of clinical
GC paraffin specimens showed that ENAH expression was significantly associated with tumor infiltration and local lymph node metastasis. These results suggest that increased expression of ENAH may further promote GC progression.

ENAH expression was previously reported to correlate with tumor grade and vascular invasion in salivary tumors [9], and is associated with tumor differentiation and clinical stage hepatocellular carcinoma [13]. Herein, in a large GC population (238 cases), we found that the expression of ENAH was significantly 
Table 3: Correlation between ENAH expression and clinicopathological variables of 155 TCGA gastric cancer cases

\begin{tabular}{|c|c|c|c|c|c|}
\hline \multirow{2}{*}{ Clinicopathological parameters } & \multirow{2}{*}{$n^{\mathrm{a}}$} & \multicolumn{2}{|c|}{ ENAH expression } & \multirow{2}{*}{$\chi^{2}$} & \multirow{2}{*}{$P$ value } \\
\hline & & High & Low & & \\
\hline All & 155 & 88 & 67 & & \\
\hline \multicolumn{6}{|l|}{ Age (years) } \\
\hline$<55$ & 31 & 25 & 6 & 8.997 & $0.003 *$ \\
\hline$\geq 55$ & 124 & 63 & 61 & & \\
\hline Gender & & & & 0.015 & 0.903 \\
\hline Male & 98 & 56 & 42 & & \\
\hline Female & 57 & 32 & 25 & & \\
\hline Tumor infiltration & & & & 5.183 & $0.023^{*}$ \\
\hline $\mathrm{T} 1-2$ & 35 & 14 & 21 & & \\
\hline T3-4 & 120 & 74 & 46 & & \\
\hline Local lymph node metastasis & & & & 0.044 & 0.834 \\
\hline N0-1 & 94 & 54 & 40 & & \\
\hline $\mathrm{N} 2-3$ & 61 & 34 & 27 & & \\
\hline Distant metastasis & & & & 0.015 & 0.903 \\
\hline M0 & 148 & 84 & 64 & & \\
\hline M1 & 7 & 4 & 3 & & \\
\hline TNM staging & & & & 1.894 & 0.595 \\
\hline 1 & 22 & 10 & 12 & & \\
\hline 2 & 54 & 30 & 24 & & \\
\hline 3 & 68 & 42 & 26 & & \\
\hline 4 & 11 & 6 & 5 & & \\
\hline
\end{tabular}

a Numbers of cases in each group. * Statistically significant $(P<0.05)$.

correlated with tumor size $(P<0.001)$, tumor infiltration $(P<0.001)$, local lymph node metastasis $(P=0.001)$, and TNM stage $(P<0.001)$. In addition, we detected higher ENAH immune reactivity in poorly differentiated GC tissues than in well-differentiated ones, suggesting that amplified ENAH expression might correlate with GC dedifferentiation. TCGA data analyses also showed that ENAH expression was significantly correlated with the depth of tumor infiltration $(P=0.023)$, further supporting our proposal that ENAH plays important roles in the progression and dedifferentiation of GC.

Importantly, Kaplan-Meier survival analyses revealed that higher expression of ENAH was significantly correlated to worse overall survival than lower ENAH expression. TCGA data analyses also support our results. Cox regression analyses further demonstrated ENAH expression as an independent prognostic factor for $\mathrm{GC}$ patients. This finding indicates that high ENAH expression might be useful for classifying GC patients with a poor prognosis and provides further evidence that ENAH may promote the progression of GC. However, it is important to note that the follow-up time used in our study was relatively short, and as such, no deaths were observed among early stage (TNM stage I) GC patients. We will continue to follow-up this cohort of patients and perform further statistical analyses on survival rates in future.

With regards to its molecular mechanism of action in tumorigenesis and progression, previous studies have shown that ENAH promotes actin polymerization at the leading edge of migrating cells [15-17]. In addition, Trono et al. found the hMENA ${ }^{11 a}$ isoform sustains cell proliferation and survival in HER2-overexpressing breast cancer cells primarily through activating the HER3/ AKT axis, and contributes to HER3-mediated resistance mechanisms to PI3K inhibitors [21]. However, the 
Table 4: Univariate and multivariate analyses of overall survival of 155 TCGA gastric cancer patients

\begin{tabular}{|c|c|c|c|c|c|c|c|}
\hline \multirow{2}{*}{ Variables } & \multirow{2}{*}{$n^{\mathrm{a}}$} & \multicolumn{3}{|c|}{ Univariate analyses } & \multicolumn{3}{|c|}{ Multivariate analyses } \\
\hline & & HR & $(95 \% \mathrm{CI})$ & $P$ value & HR & $(95 \% \mathrm{CI})$ & $P$ value \\
\hline Age (years) & & & & 0.330 & & & 0.344 \\
\hline$<55$ & 31 & 1.000 & & & 1.000 & & \\
\hline$\geq 55$ & 124 & 1.499 & $0.664-3.385$ & & 1.484 & $0.656-3.357$ & \\
\hline Gender & & & & 0.956 & & & \\
\hline Male & 98 & 1.000 & & & 1.000 & & 0.640 \\
\hline Female & 57 & 0.983 & $0.532-1.817$ & & 0.861 & $0.459-1.614$ & \\
\hline Tumor infiltration & & & & 0.064 & & & \\
\hline $\mathrm{T} 1-2$ & 35 & 1.000 & & & & & \\
\hline T3-4 & 120 & 2.279 & $0.953-5.451$ & & & & \\
\hline $\begin{array}{l}\text { Local lymph node } \\
\text { metastasis }\end{array}$ & & & & $0.049 *$ & & & \\
\hline N0-1 & 94 & 1.000 & & & & & \\
\hline $\mathrm{N} 2-3$ & 61 & 1.818 & $1.004-3.292$ & & & & \\
\hline Distant metastasis & & & & $0.002 *$ & & & \\
\hline M0 & 148 & 1.000 & & & & & \\
\hline M1 & 7 & 5.213 & $1.820-14.932$ & & & & \\
\hline TNM staging & & & & $0.042 *$ & & & $0.037 *$ \\
\hline 1 & 22 & 1.000 & & & 1.000 & & \\
\hline 2 & 54 & 1.555 & $0.503-4.804$ & & 1.433 & $0.462-4.444$ & \\
\hline 3 & 68 & 2.064 & $0.703-6.057$ & & 1.981 & $0.675-5.809$ & \\
\hline 4 & 11 & 5.245 & $1.474-18.667$ & & 5.313 & $1.474-19.156$ & \\
\hline ENAH & & & & 0.056 & & & \\
\hline Low & 67 & 1.000 & & & & & \\
\hline High & 88 & 1.914 & $0.984-3.724$ & & & & \\
\hline
\end{tabular}

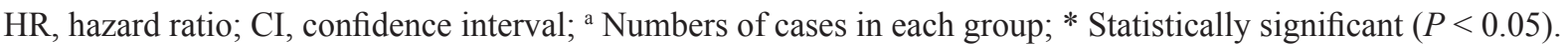

molecular mechanisms of ENAH in GC require thorough investigation in future.

In conclusion, we have demonstrated amplified expression of ENAH in gastric adenocarcinoma and its correlation with a more malignant phenotype and unfavorable prognosis in a large number of clinical GC samples. We confirmed that ENAH enhance GC cell growth, colony formation, cell migration, and invasion in vitro. Taken together, our research suggests that ENAH might serve as a candidate prognostic biomarker for GC patients and a potential target for gene therapy in the treatment of GC patients.

\section{MATERIALS AND METHODS}

\section{Ethics statement}

The research was approved by the Ethics Committee of Shandong Academy of Medical Sciences and Ethics Committee of Sun Yat-sen University Cancer Center. Written informed consent was obtained from each patient involved in the study.

\section{Cell lines and culture}

The GC cell lines MKN45, SGC7901, and MGC803 were obtained from the Committee of Type Culture 
Collection of Chinese Academy of Sciences (Shanghai, China). All cells were cultured in RPMI 1640 medium containing 10\% heat-inactive fetal bovine serum (FBS, Gibco, Grand Island, NY). The cells were incubated at $37^{\circ} \mathrm{C}$ in a humidified $5 \% \mathrm{CO}_{2}$ atmosphere.

\section{Tissue samples}

GC tissues and adjacent noncancerous gastric tissue samples were collected from 36 primary GC patients undergoing radical gastrectomy at Sun Yat-sen University Cancer Center between 2010 and 2012. None of the patients had been treated before surgery. Fresh tissues were immediately immersed in RNA later (Ambion, Inc., USA) to avoid RNA degradation, and then stored at $4^{\circ} \mathrm{C}$ overnight. All samples were subsequently frozen at $-80^{\circ} \mathrm{C}$ until RNA and protein extraction was performed.

\section{GC patients and follow-up}

Paraffin-embedded primary GC samples were obtained from 238 postoperative patients in Sun Yat-sen University Cancer Center between January 2003 and December 2006. All patients in our study belonged to the same ethnic group. The patients were selected according to the criteria: (1) histopathological identification of gastric adenocarcinoma; (2) limited or extended surgical history including gastrectomy plus lymphadenectomy; (3) no chemotherapy and radiotherapy before surgery; (4) complete follow-up data; (5) no history of other synchronous malignancies or familial malignancy; (6) no recurrent $\mathrm{GC}$ or remnant $\mathrm{GC}$; and (7) no death in the perioperative period. The surgery was performed by experienced surgeons following the Japanese Gastric Cancer Association (JGCA) guidelines.

The TCGA clinical GC data were downloaded from the UCSC Cancer Genomics Browser database (https:// genome-cancer.ucsc.edu/). The $155 \mathrm{GC}$ patients with overall follow-up data were selected according to the criteria described above.

Postoperative follow-up of 238 patients from Sun Yat-sen University Cancer Center was conducted every 3 months for the first 2 years, every 6 months during the third to fifth years, and then annually for an additional 5 years or until patient death, whichever occurred first. The characteristics of these patients are listed in Table 1. The Tumor-Node-Metastasis (TNM) stage was recorded based on the $7^{\text {th }}$ edition of the International Union Against Cancer (UICC).

\section{Real-time qRT-PCR}

Total RNA was extracted from fresh tissue samples by TRIzol Reagent (Invitrogen, Carlsbad, CA) according to manufacturer's instructions. The concentration and quality of total RNA was assessed using a Nanodrop Spectrophotometer (ND-1000; Thermo Scientific) by measuring the absorbance at $260 \mathrm{~nm}$. The cDNA was synthesized through reverse transcription (RT) with 2 $\mu \mathrm{g}$ total RNA in a $25 \mu \mathrm{L}$ reaction system using M-MLV Reverse Transcriptase (Promega, Fitchburg, WI). The reaction system was incubated at $70^{\circ} \mathrm{C}$ for $5 \mathrm{~min}, 42^{\circ} \mathrm{C}$ for $1 \mathrm{~h}$. The resulting cDNA was subjected to real-time qRT-PCR analyses to evaluate the relative mRNA levels of ENAH and GAPDH (glyceraldehyde-3-phosphate dehydrogenase, as an internal control) in primary GC tumors compared to the paired noncancerous gastric tissues.

Gene-specific amplification was carried out using an ABI 7900HT Real-time PCR system (Life Technologies, Carlsbad, CA) with a $15 \mu \mathrm{L}$ reaction system containing 0.5 $\mu \mathrm{l}$ cDNA, $7.5 \mu 12 \times$ SYBR Green master mix (Invitrogen, Carlsbad, California, USA), and $200 \mathrm{nM}$ of the appropriate oligonucleotide primers. All measurements were performed in triplicate, after undergoing the following reaction cycle: preheat at $95^{\circ} \mathrm{C}$ for $10 \mathrm{~min}, 40$ cycles of $95^{\circ} \mathrm{C}$ for $30 \mathrm{sec}$, and $60^{\circ} \mathrm{C}$ for $1 \mathrm{~min}$. The melting curve was measured at $95^{\circ} \mathrm{C}$ for $15 \mathrm{sec}, 60^{\circ} \mathrm{C}$ for $15 \mathrm{sec}$, and $72^{\circ} \mathrm{C}$ for $15 \mathrm{sec}$. The $\mathrm{Ct}$ (threshold cycle) value of each sample was measured during exponential amplification, and was calculated from threshold cycles with the software SDS 2.3. Data were analyzed using the comparative threshold cycle method $\left(2^{-\Delta C T}\right)$. Relative expression levels of ENAH were normalized to the geometric mean of the internal control GAPDH.

Primers for real-time PCR were: ENAH forward 5'-TCAAGGGTAAGGGAAACTGG-3', and reverse 5'-TGGCTCACAAGTGGTCCTCC-3'; GAPDH forward 5'-CTCCTCCTGTTCGACAGTCAGC-3', and reverse 5'-CCCAATACGACCAAATCCGTT-3'.

\section{Western blot analysis}

Total protein of cell or tissue lysate was isolated as previously described [22]. About $30 \mu \mathrm{g}$ of the protein extraction was separated by sodium dodecyl sulfate polyacrylamide gel electrophoresis and then transferred to a polyvinylidene fluoride membrane. After blocking the nonspecific binding sites for $60 \mathrm{~min}$ with $5 \%$ non-fat milk, the membrane was incubated with a rabbit monoclonal antibody against ENAH (Cell Signaling Technology, at 1000 dilution) at $4{ }^{\circ} \mathrm{C}$ overnight. Membranes were washed three times with TBST (tris buffered saline with $1 \%$ tween-20) and incubated with horseradish peroxidase (HRP)-conjugated goat anti-rabbit antibody (1:5000 dilution; AQ132P, Merck, White house Station, NJ) at $37^{\circ} \mathrm{C}$ for $1 \mathrm{~h}$.

After 3 washes, the bands were detected by an enhanced chemiluminescence system (Cell Signaling Technology, Danvers, Massachusetts, USA). The internal control GAPDH was detected using a HRP-conjugated mouse anti-human GAPDH monoclonal antibody (Shanghai Kangchen, China, at 1:5000 dilution). Band 
density was measured with ImageJ software (National Institutes of Health, Bethesda, MD) and was standardized to that of GAPDH.

\section{Expression plasmids and transient transfections}

A eukaryotic expression plasmid pDC316-mCMVEGFP, containing the full-length human Mena cDNA, was obtained from the Land Biology Company (Guangzhou, China). Empty vector was used as a negative control. GC cells were cultured in 6-well plates until they reached 85$90 \%$ confluence. Transient transfections were performed using Lipofectamine 2000 (Invitrogen), according to the manufacturer's instructions. At $48 \mathrm{~h}$ after transfection, protein expression was examined by western blotting.

\section{RNA oligonucleotides and cell transfections}

Small interfering RNA (siRNA, $20 \mu \mathrm{M}$, synthesized by GenePharma Company, Shanghai, China) was transfected into GC cells using Lipofectamine RNAi MAX (Invitrogen) according to the manufacturer's instructions. The siRNA sequences were: siRNA-ENAH\#1, sense 5'-GGUCCUAUGAUUCAUUACATT-3', and antisense 5'-UGUAAUGAAUCAUAGGACCTT-3'; '; ENAH\#2, sense 5'-GCGAGAAAGAAUGGAAAGATT-3', and antisense 5'- UCUUUCCAUUCUUUCUCGCTT-3'; negative control (NC), sense 5'-UUCUCCGAACGUGU CACGUTT-3', and antisense 5'-ACGUGACACGUUCG GAGAATT-3'. After transfection for $48 \mathrm{~h}$, protein expression was examined by western blotting.

\section{Proliferation assay}

The proliferation rate of cells was evaluated using the MTS Cell Proliferation kit (Promega, Beijing, China) according to manufacturer's instructions. Cells were seeded in 96 -well plates $\left(5 \times 10^{2}\right.$ per well), and each experiment was done in triplicate.

\section{Colony formation assay}

Cells were seeded in six-well plates $(500$ cells/ well) and cultured at $37^{\circ} \mathrm{C}$ in an atmosphere of $5 \% \mathrm{CO}_{2}$ for 10 days. Surviving colonies ( $>50$ cells per colony) were washed twice with PBS, fixed in $75 \%$ alcohol for $30 \mathrm{~min}$, and stained with $0.5 \%(\mathrm{~m} / \mathrm{v})$ crystal violet for 30 min. Colony-forming efficiency (CFE\%) was defined as the ratio of the number of colonies formed in culture to the number of cells inoculated. Three independent experiments were performed.

\section{Migration and invasion assays}

Migration and invasion assays were performed in Transwell chambers $(8-\mu \mathrm{m}$ pores, Corning, Shanghai, China) placed in 24-well plates. For invasion assay, the bottom of the Transwell chamber was coated with a thin layer of $0.5 \mathrm{mg} / \mathrm{ml}$ Matrigel Basement Membrane Matrix (BD Biosciences, Bedford, MA, USA). Cells in $150 \mu \mathrm{L}$ RPMI $1640\left(2 \times 10^{5}\right.$ cells $/ \mathrm{mL}$ for migration assay or $1 \times 10^{6}$ cells $/ \mathrm{mL}$ for invasion assay) without FBS were added into the upper Transwell chamber, and $500 \mu \mathrm{L}$ RPMI 1640 containing $20 \%$ FBS was placed in the lower chamber. The cells were incubated at $37^{\circ} \mathrm{C}$ and allowed to migrate or invade through the Matrigel layer. After $48 \mathrm{~h}$, the cells were fixed with $75 \%$ methanol for $10 \mathrm{~min}$. The migrated or invaded cells on the lower surface of Transwell chamber were stained with $0.5 \%(\mathrm{~m} / \mathrm{v})$ crystal violet for $1 \mathrm{~h}$. The stained cells were counted in 10 random fields under an inverted microscope. Each experiment was carried out in three separate wells, and independent experiments were repeated at least three times.

\section{Immunohistochemistry and semi-quantitative analysis}

Immunohistochemistry analysis was performed as previously described [22]. The antibodies were: a rabbit monoclonal antibody against ENAH (Cell Signaling Technology, at 500 dilution), and a biotinylated secondary antibody (Zhongshan Golden Bridge Biotech, Beijing, China).

The ENAH protein expression level was assessed by an immunostaining score, calculated as the sum of the percent positivity of stained tumor cells and the staining intensity, and ranged from 0 to 6 . Staining intensity was scored as: 0 (no staining), 1 (weak staining, visible at high magnification), 2 (moderate staining, visible at low magnification), and 3 (dark staining, strikingly positive at low magnification). The percentage of positive staining was scored as: 0 ( $0-9 \%$, negative), $1(10 \%-25 \%$, sporadic), 2 ( $26 \%-50 \%$, focal), or $3(51 \%-100 \%$, diffuse). ENAH expression was defined as: "-" (negative; score 0), "+" (weakly positive; score 1-2), "++" (positive; score 3-4), "+++" (strongly positive; score 5-6). Based on the ENAH expression levels, we divided the GC patients into two groups: low ENAH expression (ENAH "-." or ENAH “+”) and high ENAH expression (ENAH “++" or ENAH “+++").

The 155 TCGA patients were divided into two groups: low ENAH expression (ENAH expression value $<3.0000$ ) and high ENAH expression (ENAH expression value $\geq 3.0000$ ).

\section{Statistical analysis}

Statistical analyses were carried out with the Statistical Package for the Social Sciences, version 17.0 (SPSS Inc., Chicago, IL, USA). Differences between levels of mRNA and protein expression in tumor samples and their paired non-tumor tissue samples were assessed using the paired-sample $t$ test. ANOVA analysis was used 
to detect significant differences in the cell proliferation curves. The relationships between ENAH expression and various clinicopathological parameters were assessed using the chi-squared test. Survival curves were plotted using the Kaplan-Meier method and groups compared using the log-rank test. The Cox proportional hazard regression model was used for univariate and multivariate analyses to identify the effects of clinicopathological variables of GC and ENAH expression on survival. The results were expressed as mean \pm standard deviation (SD), and a two-sided $P$ value less than 0.05 was considered to be statistically significant.

\section{Author contributions}

DDW, BS and PW conceived and designed the experiments. DDW and QJ performed the experiments. LLW, SFH, YBC and GDS analyzed the data. SFS, SWS, $\mathrm{TW}$, and FJL contributed reagents/materials/analysis tools. DDW wrote the paper. DDW, BS, and PW provided administrative support and funded experiments. All authors read and approved the final manuscript.

\section{CONFLICTS OF INTEREST} interests.

All authors declare that they have no competing

\section{FUNDING}

This work was supported by the National Natural Science Foundation of China (grant number 31501132), The Innovation Project of Shandong Academy of Medical Sciences, The Distinguished Experts of Taishan Scholar Project (grant number ts201511074), and Shandong Key Research and Development Plan (grant number 2016GSF202042).

\section{REFERENCES}

1. Choi YY, Noh SH, Cheong JH. Evolution of Gastric Cancer Treatment: From the Golden Age of Surgery to an Era of Precision Medicine. Yonsei Med J. 2015; 56:1177-85.

2. Ferlay J, Soerjomataram I, Dikshit R, Eser S, Mathers C, Rebelo M, Parkin DM, Forman D, Bray F. Cancer incidence and mortality worldwide: sources, methods and major patterns in GLOBOCAN 2012. Int J Cancer. 2015; 136:E359-86.

3. World Health Organization (WHO) Fact sheet $N^{\circ} 297$. Updated February 2015.

4. Jung KW, Won YJ, Kong HJ, Oh CM, Cho H, Lee DH, Lee KH. Cancer statistics in Korea: incidence, mortality, survival, and prevalence in 2012. Cancer Res Treat. 2015; 47:127-41.
5. Wang YC, Wei LJ, Liu JT, Li SX, Wang QS. Comparison of Cancer Incidence between China and the USA. Cancer Biol Med. 2012; 9:128-132.

6. Apicella M, Corso S, Giordano S. Targeted therapies for gastric cancer: failures and hopes from clinical trials. Oncotarget. 2017; 8:57654-57669. https://doi.org/10.18632/ oncotarget.14825.

7. Nobili S, Bruno L, Landini I, Napoli C, Bechi P, Tonelli F, Rubio CA, Mini E, Nesi G. Genomic and genetic alterations influence the progression of gastric cancer. World J Gastroenterol. 2011; 17:290-299.

8. Celik S, Kizıltan R, Yılmaz EM, Yılmaz Ö, Demir H. Potential diagnostic and prognostic significance of plasma prolidase activity in gastric cancer. Biomark Med. 2017; 11:319-327. https://doi.org/10.2217/bmm-2016-0367.

9. Philippar U, Roussos ET, Oser M, Yamaguchi H, Kim HD, Giampieri S, Wyckoff JB, Lauffenburger DA, Sahai E, Condeelis JS, Gertler FB. A Mena invasion isoform potentiates EGF-induced carcinoma cell invasion and metastasis. Developmental cell. 2008; 15:813-28

10. Gurzu S, Krause M, Ember I, Azamfirei L, Gobel G, Feher $\mathrm{K}$, Jung I. Mena, a new available marker in tumors of salivary glands? Eur J Histochem. 2012; 56:e8.

11. Tanaka N, Yoshida H, Suzuki Y, Harigaya K. Relative expression of hMena1la and hMenaINV splice isoforms is a useful biomarker in development and progression of human breast carcinoma. Int J Oncol. 2014; 45:1921-8.

12. Wang W, Mouneimne G, Sidani M, Wyckoff J, Chen X, Makris A, Goswami S, Bresnick AR, Condeelis JS. The activity status of cofilin is directly related to invasion, intravasation, and metastasis of mammary tumors. The Journal of cell biology. 2006; 173:395-404.

13. Di Modugno F, Bronzi G, Scanlan MJ, Del Bello D, Cascioli S, Venturo I, Botti C, Nicotra MR, Mottolese M, Natali PG, Santoni A, Jager E, Nisticò P. Human Mena protein, a serex-defined antigen overexpressed in breast cancer eliciting both humoral and CD8+ T-cell immune response. Int J Cancer. 2004; 109:909-918.

14. Hu K, Wang J, Yao Z, Liu B, Lin Y, Liu L, Xu L. Expression of cytoskeleton regulatory protein Mena in human hepatocellular carcinoma and its prognostic significance. Med Oncol. 2014; 31:939.

15. Bear JE, Loureiro JJ, Libova I, Fässler R, Wehland J, Gertler FB. Negative regulation of fibroblast motility by Ena/ VASP proteins. Cell. 2000; 101:717-728.

16. Bear JE, Svitkina TM, Krause M, Schafer DA, Loureiro JJ, Strasser GA, Maly IV, Chaga OY, Cooper JA, Borisy GG, Gertler FB. Antagonism between Ena/VASP proteins and actin filament capping regulates fibroblast motility. Cell. 2002; 109:1-20.

17. Boëda B, Briggs DC, Higgins T, Garvalov BK, Fadden AJ, McDonald NQ, Way M. Tes, a specific Mena interacting partner, breaks the rules for EVH1 binding. Mol Cell. 2007; 28:1071-82. 
18. Gertler F, Condeelis J. Metastasis: tumor cells becoming MENAcing. Trends Cell Biol. 2011; 21:81-90.

19. Di Modugno F, DeMonte L, Balsamo M, Bronzi G, Nicotra MR, Alessio M, Jager E, Condeelis JS, Santoni A, Natali PG, Nisticò P. Molecular cloning of hMena (ENAH) and its splice variant $\mathrm{hMena}^{+11 \mathrm{a}}$ : epidermal growth factor increases their expression and stimulates hMena ${ }^{+11 a}$ phosphorylation in breast cancer cell lines. Cancer Res. 2007; 67:2657-2665.

20. Urbanelli L, Massini C, Emiliani C, Orlacchio A, Bernardi G, Orlacchio A. Characterization of human Enah gene. Biochim Biophys Acta. 2006; 1759:99-107.
21. Trono P, Di Modugno F, Circo R, Spada S, Di Benedetto A, Melchionna R, Palermo B, Matteoni S, Soddu S, Mottolese M, De Maria R, Nisticò P. hMENA(11a) contributes to HER3-mediated resistance to PI3K inhibitors in HER2overexpressing breast cancer cells. Oncogene. 2016; 35:887-96.

22. Li YF, Wang DD, Zhao BW, Wang W, Huang CY, Chen YM, Zheng Y, Keshari RP, Xia JC, Zhou ZW. High level of COP1 expression is associated with poor prognosis in primary gastric cancer. Int J Biol Sci. 2012; 8:1168-77. 